\title{
AVALIAÇÃO REGIONAL DE CULTIVARES DE SOJA NO NOROESTE PAULISTA - SAFRAS 2011/12 E 2012/13
}

\author{
BORGES, Wander Luis Barbosa ${ }^{1}$ \\ MATEUS, Gustavo Pavan'; \\ FREITAS, Rogério Soares de ${ }^{1}$; \\ LAZARINI, Edson ${ }^{3}$; \\ CAZENTINI FILHO, Gerson ${ }^{4}$; \\ HIPÓLITO, Jorge Luiz'; \\ TOKUDA, Flávio Sueo ${ }^{6}$; \\ TOMAZINI, Nicola Roberto ${ }^{7}$; \\ GASPARINO, Adriano Custódio ${ }^{6}$; \\ FINOTO, Everton Luis
}

ISSUE DOI: $10.3738 /$ nucleus.v0i0.915

RESUMO: Na safra 2012/13, o Brasil deverá ser o maior produtor e exportador mundial de soja, posições anteriormente ocupadas pelos Estados Unidos. O trabalho foi desenvolvido em Votuporanga, SP, Araçatuba, SP e Pontes Gestal, SP, durante a safra de 2011/12, e em Andradina, SP, Araçatuba, SP, Riolândia, SP, Votuporanga, SP e Selvíria, MS, localizada a 13,9 km de Ilha Solteira, SP, na safra 2012/13, e teve por objetivo avaliar a adaptação de diferentes cultivares de soja, as condições edafoclimáticas do Noroeste Paulista. $\mathrm{O}$ delineamento experimental utilizado foi o de blocos casualizados com 18 tratamentos no primeiro ano e 25 no segundo e três repetições. As parcelas experimentais foram compostas por quatro linhas de $5 \mathrm{~m}$, com espaçamento de $0,45 \mathrm{~m}$ entre linhas. Os tratamentos constaram das seguintes cultivares na safra 2011/12: Embrapa 48; BRSMG 68 (Vencedora); BRS Valiosa RR; CD 208; CD 219 RR; NS 7100 RR; NA 5909 RG; NA 7255 RR; RA 626 RR; RA 518 RR; M-Soy 7908 RR; SYN 1080 RR; SYN 2395 RR; SYN 9078 RR; P 98Y11 RR; P $98 Y 12$ RR; BMX Potência RR; DOW 5D711 RR, e das seguintes cultivares na safra 2012/13: BRS 232; BRS 284; BRS Valiosa RR; CD 208; P 98 Y11 RR; P 98Y12 RR; P 97R01; SYN 1163 RR; SYN 1281 RR; SYN 1283 RR; SYN 9078 RR; NA 5909 RG; NA 7200 RR; NS 7255 RR; NS 7490 RR; NS 7901 RR; Fundacep 66 RR; FPS Paranapanema RR; FPS Iguaçu RR; INT 6100 RR; INT 6201 RR; BMX Potência RR; BMX Desafio RR; Anta 82 RR; M-Soy 7908 RR. Devido a grande variabilidade dos resultados, é necessária a continuação dos estudos para melhor recomendação das cultivares mais adaptadas a Região Noroeste Paulista.

Palavras-chave: Glycine $\max ($ L.) Merrill, Estabilidade de produção, Características agronômicas.

\section{REGIONAL EVALUATION OF SOYBEAN CULTIVARS IN THE NORTHWEST OF SÃO PAULO STATE, BRAZIL - CROP YEARS 2011/12 AND 2012/13}

\footnotetext{
${ }^{1}$ Pesquisador Científico, Dr. - APTA - PRDTA Noroeste Paulista, Votuporanga, SP;

${ }^{2}$ Pesquisador Científico, Dr. - APTA - PRDTA Extremo Oeste, Andradina, SP;

${ }^{3}$ Professor, Dr. - UNESP - FEIS - DFTASE, Ilha Solteira, SP;

${ }^{4}$ Assistente Agropecuário - CATI - DSMM - NPS, Fernandópolis, SP;

${ }^{5}$ Assistente Agropecuário - CATI - EDR Araçatuba, Araçatuba, SP;

${ }^{6}$ Assistente Agropecuário - CATI - EDR Votuporanga, Votuporanga, SP;

${ }^{7}$ Assistente Agropecuário - CATI - DSMM - NPS, Araçatuba, SP;

${ }^{8}$ Pesquisador Científico, Dr. - APTA - PRDTA Centro Norte, Pindorama, SP.
}

SUMMARY: In the crop year 2012/13, Brazil will be the largest producer and exporter of soybeans, positions previously occupied by the U.S. The study was conducted in Votuporanga, SP, Araçatuba, SP and Pontes Gestal, SP, during the crop year 2011/12, and Andradina, SP, Araçatuba, SP, Riolândia, SP, Votuporanga, SP and Selvíria, MS, located 13,9 km of Ilha Solteira, SP, in 2012/13 and aimed to evaluate the suitability of different soybean cultivars, soil and climatic conditions of the Northwest of São Paulo State. The experimental design was a randomized block with 18 treatments in the first year and 25 in the second and three replications. The plots were composed of four lines of $5 \mathrm{~m}$, with $0,45 \mathrm{~m}$ spacing between lines. Treatments consisted of the following cultivars in crop year 2011/12: Embrapa 48; BRSMG 68 (Vencedora); BRS Valiosa RR; CD 208; CD 219 RR; NS 7100 RR; NA 5909 RG; NA 7255 RR; RA 626 RR; RA 518 RR; M-Soy 7908 RR; SYN 1080 RR; SYN 2395 RR; SYN 9078 RR; P $98 Y 11$ RR; P 98Y12 RR; BMX Potência RR; DOW 5D711 RR, and the following cultivars in 2012/13: BRS 232; BRS 284; BRS 
Valiosa RR; CD 208; P 98Y11 RR; P 98Y12 RR; P 97R01; SYN 1163 RR; SYN 1281 RR; SYN 1283 RR; SYN 9078 RR; NA 5909 RG; NA 7200 RR; NS 7255 RR; NS 7490 RR; NS 7901 RR; Fundacep 66 RR; FPS Paranapanema RR; FPS Iguaçu RR; INT 6100 RR; INT 6201 RR; BMX Potência RR; BMX Desafio RR; Anta 82 RR; M-Soy 7908 RR. Due to the great variability of the results, it is necessary to further study for better recommendation of cultivars adapted to the Northwest Region of São Paulo State.

Keywords: Glycine $\max ($ L.) Merrill, Production stability, Agronomic characteristics.

\section{INTRODUÇÃO}

Na safra 2012/13, o Brasil deverá ser o maior produtor e exportador mundial de soja, posições anteriormente ocupadas pelos Estados Unidos (PEREIRA, 2013).

As áreas de produção nacional de soja, nas safras 2011/12 e 2012/13 (dados estimados), foram de 25,042 e 27,715 milhões de hectares, respectivamente, com uma produção de grãos de 66,383 milhões de toneladas, na safra 2011/12 e de 81,513 milhões de toneladas na safra 2012/13 (dados estimados), participando da produção brasileira de grãos com 44,29\%, que está estimada em 184,04 milhões de toneladas (CONAB, 2013).

Cultivares melhoradas, portadoras de genes capazes de expressar alta produtividade, ampla adaptação e boa resistência/tolerância a fatores bióticos ou abióticos adversos, representam usualmente uma das mais significativas contribuições à eficiência do setor produtivo. $\mathrm{O}$ ganho genético proporcionado pelas novas cultivares ao setor produtivo tem sido muito significativo, cerca de $1,38 \%$ ao ano (EMBRAPA, 2011).

A cada ano as empresas de melhoramento criam novas cultivares para atender as demandas dos produtores que tem diante de si a necessidade cada vez maior de produzir mais, apesar das dificuldades. Saber escolher a combinação de cultivares que irá resultar na melhor produtividade é tarefa importante e depende do conhecimento profundo dos fatores restritivos presentes na área a ser explorada. Além disso, o conhecimento das características das cultivares e de suas interações com os fatores de produção, formará a base de informações que irá determinar as melhores cultivares para cada área de cultivo (FUNDAÇÃO MT, 2007).

Segundo Peixoto et al. (2000), existe grande variabilidade entre cultivares de soja com relação a sensibilidade a época e local de semeadura, e de acordo com Komori et al. (2004), muitas cultivares estão disponíveis no mercado apresentando grande diversificação, principalmente quanto à interação genótipo $\mathrm{x}$ ambiente e, por esse motivo, é desejável que os produtores tenham conhecimentos mais aprofundados das cultivares disponíveis em diferentes ambientes.

Assim, as avaliações regionais são de suma importância, pois fomentam os sojicultores na escolha dos materiais mais adaptados as condições de clima e de solo de determinada região.

O presente trabalho teve por objetivo avaliar a adaptação de diferentes cultivares de soja, as condições edafoclimáticas do Noroeste Paulista.

\section{MATERIAL E MÉTODOS}

Na safra 2011/12 o trabalho foi desenvolvido em Votuporanga, SP, no Polo Regional de Desenvolvimento Tecnológico dos Agronegócios (PRDTA) do Noroeste Paulista - APTA, e em Araçatuba, SP e Pontes Gestal, SP, em propriedades particulares. 
Na safra 2012/13 o trabalho foi desenvolvido em Votuporanga, SP, no PRDTA do Noroeste Paulista - APTA, em Andradina, SP, no PRDTA do Extremo Oeste - APTA, em Selvíria, MS, localizada a 13,9 km de Ilha Solteira, SP, na Fazenda de Ensino, Pesquisa e Extensão da Faculdade de Engenharia de Ilha Solteira (FEIS)/UNESP e em Araçatuba, SP e Riolândia, SP, em propriedades particulares.

O clima da região, segundo a classificação de Köppen, é do tipo Aw, definido como tropical úmido com estação chuvosa no verão e seca no inverno, apresentando temperatura média anual de $24,5^{\circ} \mathrm{C}$, precipitação média anual de $1.232 \mathrm{~mm}$, umidade relativa média anual de 64,8\% e déficit hídrico acentuado nos meses de junho a setembro (HERNANDEZ et al., 1995).

O delineamento experimental utilizado foi blocos casualizados com 18 tratamentos no primeiro ano e 25 no segundo e três repetições. As parcelas experimentais foram compostas por quatro linhas de 5 m, utilizando-se as duas linhas centrais para as avaliações agronômicas.

Os tratamentos constaram das seguintes cultivares na safra 2011/12: Embrapa 48; BRSMG 68 (Vencedora); BRS Valiosa RR; CD 208; CD 219 RR; NS 7100 RR; NA 5909 RG; NA 7255 RR; RA 626 RR; RA 518 RR; M-Soy 7908 RR; SYN 1080 RR; SYN 2395 RR; SYN 9078 RR; P 98Y11 RR; P $98 Y 12$ RR; BMX Potência RR; DOW 5D711 RR, e das seguintes cultivares na safra 2012/13: BRS 232; BRS 284; BRS Valiosa RR; CD 208; P 98Y11 RR; P 98Y12 RR; P 97R01; SYN 1163 RR; SYN 1281 RR; SYN 1283 RR; SYN 9078 RR; NA 5909 RG; NA 7200 RR; NS 7255 RR; NS 7490 RR; NS 7901 RR; Fundacep 66 RR; FPS Paranapanema RR; FPS Iguaçu RR; INT 6100 RR; INT 6201 RR; BMX Potencia RR; BMX Desafio RR; Anta 82 RR; M-Soy 7908 RR.

Na safra 2011/12 o espaçamento utilizado foi de $0,45 \mathrm{~m}$ entre linhas, nos três locais, e a semeadura foi realizada no dia 01 de novembro de 2011, em Araçatuba, SP, no dia 22 de dezembro de 2011, em Pontes Gestal, SP e no dia 12 de dezembro de 2011, em Votuporanga, SP.

$\mathrm{Na}$ adubação de semeadura foi utilizado $200 \mathrm{~kg} \mathrm{ha}^{-1}$ do adubo formulado 04-20-20, em Pontes Gestal, SP e $300 \mathrm{~kg} \mathrm{ha}^{-1}$ do adubo formulado 04-2020, em Votuporanga, SP.

Na safra 2012/13 a semeadura foi realizada no dia 20 de dezembro, em Andradina, SP; no dia 13 de novembro de 2012 e se utilizou $165 \mathrm{~kg} \mathrm{ha}^{-1}$ do adubo formulado organomineral 02-07-08, em Araçatuba, SP; no dia 21 de novembro de 2012 e se utilizou $260 \mathrm{~kg} \mathrm{ha}^{-1}$ do adubo formulado 08-32-12 e $100 \mathrm{~kg} \mathrm{ha}^{-1}$ de cloreto de potássio, em Riolândia, SP; no dia 28 de novembro de 2012 e se utilizou $250 \mathrm{~kg}$ $\mathrm{ha}^{-1}$ do adubo formulado 08-28-16, em Selvíria, MS; no dia 12 de dezembro, e se utilizou $300 \mathrm{~kg} \mathrm{ha}^{-1}$ do adubo formulado 04-20-20, em Votuporanga, SP. Os espaçamentos utilizados foram: Andradina, SP: 0,45 m, Araçatuba, SP: 0,45 m, Riolândia, SP: 0,50 m, Votuporanga, SP: 0,50 m, Selvíria, MS: 0,45 m.

Na safra 2011/12, nos três locais, e na safra 2012/13, em Araçatuba, SP, Riolândia, SP e Votuporanga, SP, a semeadura foi realizada mecanicamente com semeadora de parcelas, em sistema de semeadura direta, após a adubação de semeadura, feita mecanicamente com semeadoras de grãos, e em Andradina, SP e Selvíria, MS, manualmente, abrindo-se sulcos de plantio com enxadas adaptadas, distribuindo as sementes no sulco, e depois se tampando o sulco com enxadas adaptadas. Quinze dias após a semeadura foi realizado o desbaste de plantas, deixando-se a população inicial recomendada pelos detentores de cada cultivar.

Realizou-se todo o controle fitossanitário adequado para o bom desenvolvimento da cultura da soja, em todos os locais e nas duas safras.

Os parâmetros avaliados na cultura da soja foram: altura de inserção da primeira vagem, altura de plantas e número de vagens planta ${ }^{-1}$, amostrando-se dez plantas de cada parcela, e estande final ha ${ }^{-1}$, massa de cem grãos e produtividade de grãos ha ${ }^{-1}$, nas duas linhas centrais de cada parcela. As avaliações foram 
realizadas no momento da colheita. A produtividade foi obtida padronizando-se a umidade dos grãos para $13 \%$.

Também se avaliou o grau de acamamento, utilizando-se o critério de notas de 1 a 5 , sendo 1 : todas as plantas eretas; 2: todas as plantas levemente inclinadas ou pouco acamadas; 3: todas as plantas moderadamente inclinadas ou $25 \%$ a $30 \%$ das plantas acamadas; 4 : todas as plantas bastante inclinadas ou $50 \%$ a $80 \%$ das plantas acamadas; 5 : todas ou mais de $80 \%$ das plantas acamadas.

Os dados de altura de inserção da primeira vagem, altura de plantas, grau de acamamento, número de vagens planta $^{-1}$, massa de cem grãos e produtividade de grãos foram submetidos ao teste $\mathrm{F}$ e realizado o teste Scott-Knott ( $\mathrm{p}<0,05)$, para comparação das médias.

Realizou-se a análise conjunta dos valores médios da altura de inserção da primeira vagem, altura de plantas, estande final, número de vagens, massa de cem grãos e produtividade de grãos, de dez cultivares que foram utilizadas nos três locais, na safra 2011/12, e dos valores médios da altura de inserção da primeira vagem, altura de plantas, estande final, número de vagens e produtividade de grãos de vinte e duas cultivares de soja, que não apresentaram problemas de germinação, em Andradina, SP, Riolândia, SP, Selvíria, MS e Votuporanga, SP, na safra 2012/13.

Considerou-se o estádio R2 para avaliação do florescimento e o período compreendido entre a semeadura e a colheita, para avaliação do ciclo das cultivares.

Não se comparou as médias dos estandes finais por variarem de acordo com a recomendação do detentor da cultivar.

\section{RESULTADOS E DISCUSSÃO}

Na safra 2011/12, as cultivares avaliadas apresentaram produtividade de grãos variando de 1768 a $4474 \mathrm{~kg} \mathrm{ha}^{-1}$ (Tabelas 1, 2 e 3), no entanto, não diferiram entre si, ao nível de 5\% de probabilidade, em nenhum local, já Cruz et al. (2010) encontraram diferenças significativas entre as cultivares para as variáveis número total de vagens, número total de grãos, massa de mil grãos e produtividade de grãos, e enfatizaram que isso mostra que os genótipos apresentaram características agronômicas bastante diferenciadas.

Em Araçatuba, SP e Pontes Gestal, SP, todas as cultivares tiveram produtividade de grãos superior a produtividade média nacional da cultura da soja, na safra 2011/12, que foi de $2651 \mathrm{~kg} \mathrm{ha}^{-1}$ (CONAB, 2013), sendo que em Araçatuba, SP, a cultivar SYN 2395 RR apresentou a maior massa de cem grãos, e em Pontes Gestal, SP, a cultivar BRSMG 68 (Vencedora) a menor (Tabelas 1 e 2).

A produtividade de grãos da cultivar BRSMG 68 (Vencedora) variou de 2732 a $3274 \mathrm{~kg} \mathrm{ha}^{-1}$, em Pontes Gestal, SP e Votuporanga, SP, respectivamente (Tabelas 2 e 3), inferior a produtividade média encontrada por Bighi et al. (2006), em Ipameri, GO e Jaboticabal, SP, que foi de $4303 \mathrm{~kg} \mathrm{ha}^{-1}$, e a produtividade da cultivar M-Soy 7908 RR variou de 1994 a $3522 \mathrm{~kg} \mathrm{ha}^{-1}$, em Votuporanga, SP e Araçatuba, SP, respectivamente, mostrando a variabilidade das cultivares entre os diferentes locais, e corroborando com Yuyama (1991), que enfatizou que o sucesso em relação a produtividade da cultivar é condicionado pelo genótipo do material e pela sua interação com as variações ambientais.

A cultivar NA 7255 RR apresentou produtividade de grãos, nos três locais, superiores a encontrada por Soares et al. (2012), em Lavras, MG, na mesma safra, que foi de $2002 \mathrm{~kg} \mathrm{ha}^{-1}$, e a cultivar NA 5909 RG, nas três localidades, teve produtividades superiores a encontrada por Bertagnolli et al. (2012), em Passo Fundo, RS, na mesma safra, que foi de $1809 \mathrm{~kg} \mathrm{ha}^{-1}$, conforme Tabelas 1, 2 e 3. 
A produtividade de grãos da cultivar CD 219 RR, em Araçatuba, SP e Votuporanga, SP (Tabelas 1 e 3), foram superiores a encontrada por Peluzio et al. (2010), que foram de 3281 e $2028 \mathrm{~kg} \mathrm{ha}^{-1}$, em Palmas, TO e Gurupi, TO, respectivamente.

Na safra 2012/13, as cultivares avaliadas apresentaram produtividade de grãos variando de 1643 a $5293 \mathrm{~kg} \mathrm{ha}^{-1}$, no entanto, não diferiram entre si, ao nível de $5 \%$ de probabilidade, em nenhum local (Tabelas 4, 5, 6, 7 e 8).

Algumas cultivares apresentaram problemas de germinação, oque comprometeu o estande e pode ter interferido na produtividade de grãos.

A produtividade de grãos da cultivar BMX Potência RR, em todos os locais (Tabelas 4, 5, 6, 7 e 8), foi inferior a encontrada por Hayashi et al. (2012), na safra 2010/11, em Selvíria, MS, que foi de 3712 $\mathrm{kg} \mathrm{ha}^{-1}$, por outro lado, a produtividade da cultivar Anta $82 \mathrm{RR}$, em Riolândia, SP e Selvíria, MS (Tabelas 6 e 7), foram superiores a encontra pelos mesmos autores, que foi de $3130 \mathrm{~kg} \mathrm{ha}^{-1}$.

Em Araçatuba, SP (Tabela 5), todas as cultivares tiveram produtividade de grãos superior a produtividade média nacional da cultura da soja, na safra 2012/13, que foi de $2941 \mathrm{~kg} \mathrm{ha}^{-1}$ (dados estimados) (CONAB, 2013).

A produtividade de grãos das cultivares P 98 Y11 RR e BRS Valiosa RR, em Selvíria, MS (Tabela 7), foram superiores a encontrada por Araujo Junior et al. (2012), na mesma localidade, na safra 2010/11, que foi de 3082 e $2933 \mathrm{~kg} \mathrm{ha}^{-1}$, para as cultivares P 98Y11 RR e BRS Valiosa RR, respectivamente, por outro lado, as produtividades destas cultivares, em Riolândia, SP (Tabela 6), foram inferiores a encontrada pelos mesmos autores.

É importante se considerar as condições onde foram realizados os demais estudos citados neste trabalho, pois segundo Mauad et al. (2010), a produtividade da cultura é definida pela interação entre planta, ambiente de produção e manejo.

A análise conjunta dos dados, na safra 2011/12 (Tabela 9) e na safra 2012/13 (Tabela 10), não refletiu diferenças significativas entre as cultivares avaliadas, ao nível de $5 \%$ de probabilidade, em relação a produtividade de grãos, devido a grande variabilidade que as cultivares apresentaram, refletido pelo elevado coeficiente de variação $(\mathrm{CV})$, sendo necessária a continuação dos estudos para a recomendação das cultivares mais adaptadas a Região Noroeste Paulista. 
Tabela 1. Valores médios da altura de inserção da primeira vagem, altura de plantas, grau de acamamento, estande final, número de vagens, massa de cem grãos e produtividade de grãos de diferentes cultivares de soja, Araçatuba, SP, safra 2011/12.

\begin{tabular}{|c|c|c|c|c|c|c|c|}
\hline Cultivar & $\begin{array}{c}\text { Altura de } \\
\text { inserção (cm) }\end{array}$ & $\begin{array}{c}\text { Altura de plantas } \\
\text { (cm) }\end{array}$ & Acamamento & $\begin{array}{l}\text { Estande } \\
\text { final ha }\end{array}$ & $\begin{array}{c}\text { Número de } \\
\text { vagens planta }^{-1}\end{array}$ & $\begin{array}{l}\text { Massa de } \\
\text { cem grãos }\end{array}$ & $\begin{array}{c}\text { Produtividade } \\
\qquad\left(\mathrm{kg} \mathrm{ha}^{-1}\right)\end{array}$ \\
\hline CD 208 & $17,3 \mathrm{~b}$ & $76,7 \mathrm{c}$ & 0 & 208813 & $57 \mathrm{a}$ & $14,8 \mathrm{~d}$ & 4474 \\
\hline BMX Potência RR & $19,1 \mathrm{~b}$ & $109,9 \mathrm{~b}$ & 0 & 286664 & $39 \mathrm{~b}$ & $16,7 \mathrm{~b}$ & 4171 \\
\hline SYN 1080 RR & 20,7 a & $83,9 \mathrm{c}$ & 0 & 387667 & $50 \mathrm{a}$ & $16,1 \mathrm{c}$ & 4137 \\
\hline RA 626 RR & $16,4 \mathrm{~b}$ & $84,5 \mathrm{c}$ & 0 & 211924 & $38 \mathrm{~b}$ & $16,4 \mathrm{c}$ & 3792 \\
\hline NA 7255 RR & $19,3 \mathrm{a}$ & $107,0 \mathrm{~b}$ & 0 & 280738 & $50 \mathrm{a}$ & $15,7 \mathrm{c}$ & 3740 \\
\hline SYN 9078 RR & 23,9 a & 135,4 a & 0 & 353848 & $62 \mathrm{a}$ & $15,9 \mathrm{c}$ & 3658 \\
\hline SYN 2395 RR & $15,4 \mathrm{~b}$ & $70,4 \mathrm{c}$ & 0 & 190813 & $31 \mathrm{~b}$ & $17,4 \mathrm{~b}$ & 3643 \\
\hline M-Soy 7908 RR & $23,8 \mathrm{~b}$ & $76,0 \mathrm{c}$ & 0 & 221850 & $38 \mathrm{~b}$ & 18,5 a & 3522 \\
\hline NA 5909 RG & $17,7 \mathrm{~b}$ & $67,0 \mathrm{c}$ & 0 & 188146 & $36 \mathrm{~b}$ & $17,2 \mathrm{~b}$ & 3508 \\
\hline BRS Valiosa RR & 20,2 a & $93,5 \mathrm{c}$ & 0 & 252738 & $70 \mathrm{a}$ & $16,0 \mathrm{c}$ & 2984 \\
\hline $\mathrm{CV}(\%)$ & 16,11 & 11,86 & & & 25,62 & 4,23 & 12,76 \\
\hline
\end{tabular}

Médias seguidas de mesma letra não diferem entre si pelo teste Scott-Knott a 5\% de probabilidade. 
Tabela 2. Valores médios da altura de inserção da primeira vagem, altura de plantas, grau de acamamento, estande final, número de vagens, massa de cem grãos e produtividade de grãos de diferentes cultivares de soja, Pontes Gestal, SP, safra 2011/12.

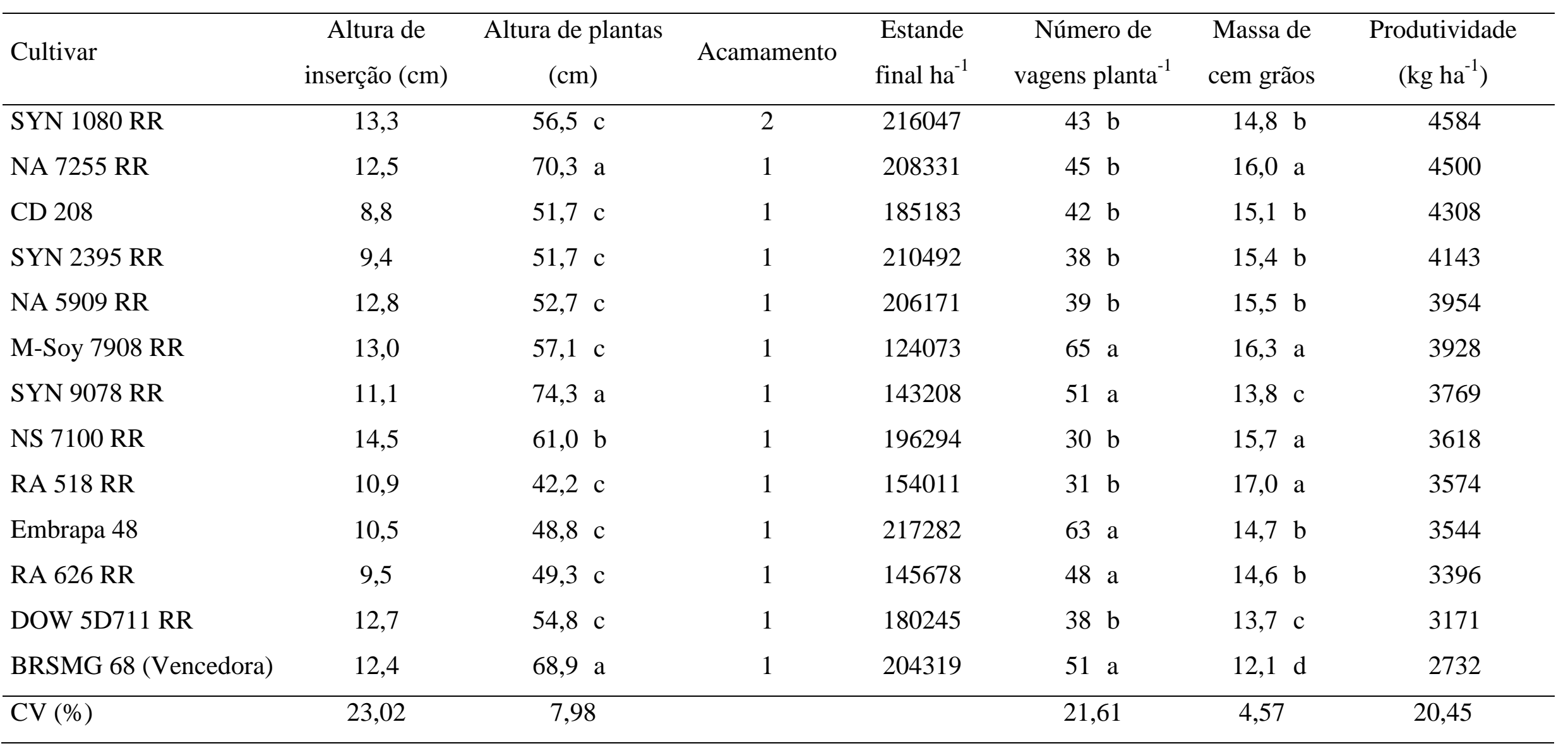

Médias seguidas de mesma letra não diferem entre si pelo teste Scott-Knott a 5\% de probabilidade. 
Tabela 3. Valores médios do ciclo, altura de inserção da primeira vagem, altura de plantas, grau de acamamento, estande final, número de vagens, massa de cem grãos e produtividade de grãos de diferentes cultivares de soja, Votuporanga, SP, safra 2011/12.

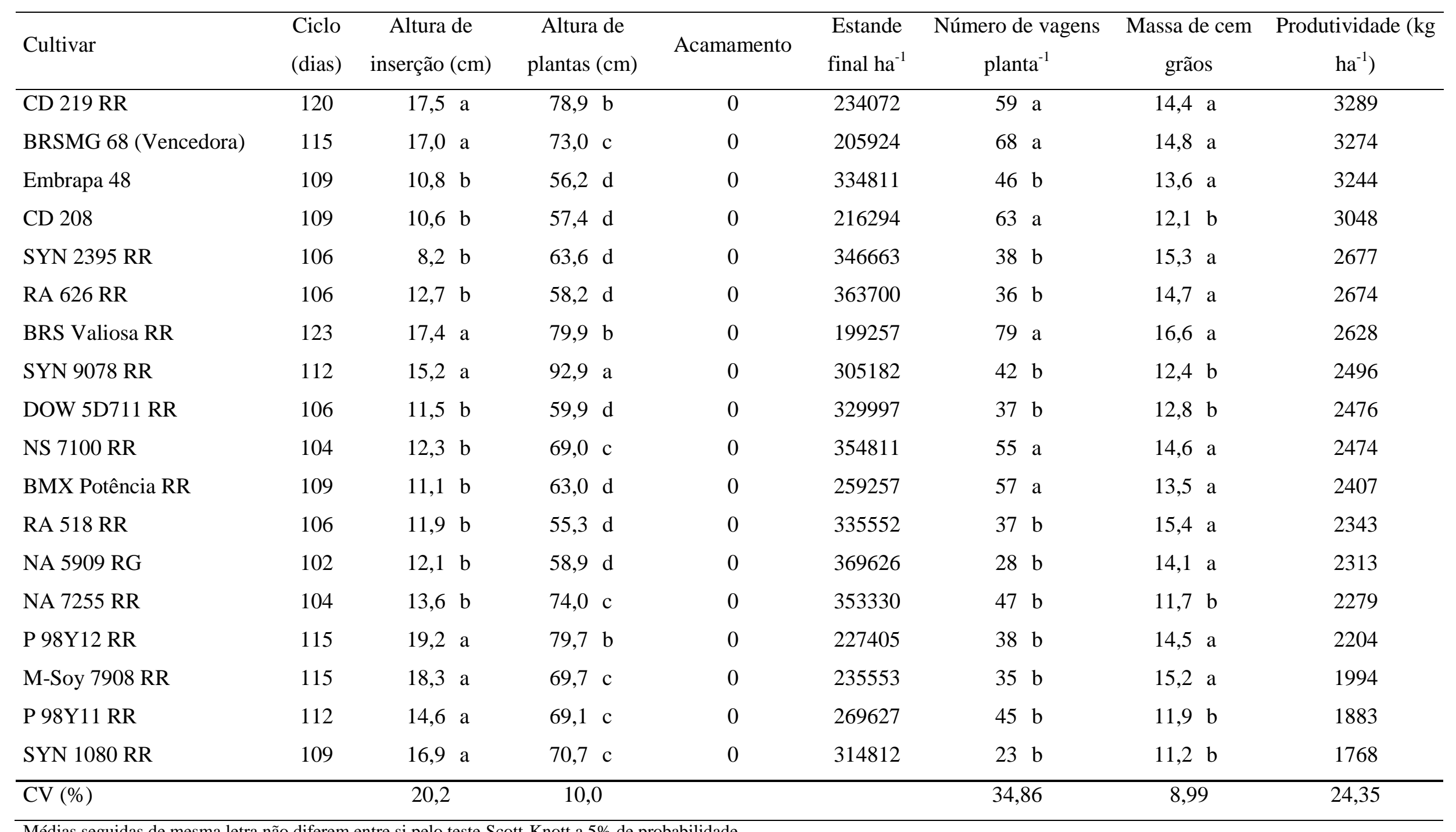

Médias seguidas de mesma letra não diferem entre si pelo teste Scott-Knott a 5\% de probabilidade. 
Tabela 4. Valores médios do ciclo, altura de inserção da primeira vagem, altura de plantas, estande final, massa de cem grãos e produtividade de grãos de diferentes cultivares de soja, Andradina, SP, safra 2012/13.

(Continua)

\begin{tabular}{|c|c|c|c|c|c|c|c|}
\hline Cultivar & $\begin{array}{l}\text { Ciclo } \\
\text { (dias) }\end{array}$ & $\begin{array}{l}\text { Altura de inserção } \\
\qquad(\mathrm{cm})\end{array}$ & $\begin{array}{l}\text { Altura de plantas } \\
\qquad(\mathrm{cm})\end{array}$ & $\begin{array}{l}\text { Estande } \\
\text { final } \mathrm{ha}^{-1}\end{array}$ & $\begin{array}{l}\text { Número de vagens } \\
\text { planta }^{-1}\end{array}$ & $\begin{array}{l}\text { Massa de } \\
\text { cem grãos }\end{array}$ & $\begin{array}{l}\text { Produtividade } \\
\qquad\left(\mathrm{kg} \mathrm{ha}^{-1}\right)\end{array}$ \\
\hline SYN 1283 RR & 121 & $10,3 \mathrm{c}$ & $69,3 \mathrm{~b}$ & 121667 & $104 \mathrm{~b}$ & $13,7 \mathrm{c}$ & 3657 \\
\hline BRS Valiosa RR & 121 & $20,0 \mathrm{~b}$ & 79,0 a & 125000 & $167 \mathrm{a}$ & $13,8 \mathrm{c}$ & 3410 \\
\hline M-Soy 7908 RR & 118 & $20,3 \mathrm{~b}$ & 76,9 a & 121667 & $82 \mathrm{~b}$ & $12,5 \mathrm{c}$ & 3293 \\
\hline NS 7901 RR & 127 & $18,4 \mathrm{~b}$ & 94,4 a & 160000 & $157 \mathrm{a}$ & $13,0 \mathrm{c}$ & 3097 \\
\hline CD 208 & 121 & $13,3 \mathrm{c}$ & $67,0 \mathrm{~b}$ & 166667 & $95 \mathrm{~b}$ & $12,4 \mathrm{c}$ & 3067 \\
\hline SYN $1281 \mathrm{RR}$ & 124 & 26,2 a & 83,7 a & 421667 & $40 \mathrm{~b}$ & $11,9 \mathrm{c}$ & 2917 \\
\hline SYN 1163 RR & 118 & $13,1 \mathrm{c}$ & 82,6 a & 161667 & $122 \mathrm{a}$ & $14,2 \mathrm{c}$ & 2883 \\
\hline BRS 232 & 118 & $7,1 \mathrm{c}$ & $50,4 \mathrm{c}$ & 149444 & $81 \mathrm{~b}$ & 22,2 a & 2837 \\
\hline NS 7490 RR & 121 & $14,0 \mathrm{c}$ & 79,0 a & 156667 & $92 \mathrm{~b}$ & $10,7 \mathrm{c}$ & 2803 \\
\hline BRS 284 & 118 & $12,1 \mathrm{c}$ & $67,8 \mathrm{~b}$ & 126667 & $125 \mathrm{a}$ & $12,2 \mathrm{c}$ & 2740 \\
\hline NS 7255 RR & 127 & $13,6 \mathrm{c}$ & 75,9 a & 190000 & $73 \mathrm{~b}$ & $11,5 \mathrm{c}$ & 2687 \\
\hline P 97R01 RR & 118 & $19,2 \mathrm{~b}$ & $67,0 \mathrm{~b}$ & 146667 & $71 \mathrm{~b}$ & $11,6 \mathrm{c}$ & 2687 \\
\hline INT 6100 RR & 118 & $10,0 \mathrm{c}$ & 72,7 a & 105000 & $90 \mathrm{~b}$ & $12,0 \mathrm{c}$ & 2680 \\
\hline BMX Potência RR & 124 & $12,2 \mathrm{c}$ & 76,1 a & 130000 & $103 \mathrm{~b}$ & $11,6 \mathrm{c}$ & 2637 \\
\hline P 98Y11 RR & 124 & $17,1 \mathrm{~b}$ & $60,8 \mathrm{~b}$ & 113333 & $108 \mathrm{~b}$ & $12,8 \mathrm{c}$ & 2610 \\
\hline Anta 82 RR & 121 & $13,8 \mathrm{c}$ & $63,2 \mathrm{~b}$ & 251667 & $52 \mathrm{~b}$ & $10,1 \mathrm{c}$ & 2543 \\
\hline
\end{tabular}


Tabela 4. Valores médios do ciclo, altura de inserção da primeira vagem, altura de plantas, estande final, massa de cem grãos e produtividade de grãos de diferentes cultivares de soja, Andradina, SP, safra 2012/13.

(Conclusão)

\begin{tabular}{|c|c|c|c|c|c|c|c|}
\hline Fundacep 66 RR & 118 & $14,3 \mathrm{c}$ & 77,0 a & 220000 & $55 \mathrm{~b}$ & $17,0 \mathrm{~b}$ & 2540 \\
\hline BMX Desafio RR & 124 & $11,1 \mathrm{c}$ & $65,8 \mathrm{~b}$ & 203333 & $81 \mathrm{~b}$ & $14,2 \mathrm{c}$ & 2537 \\
\hline FPS Paranapanema RR & 118 & $12,0 \mathrm{c}$ & $55,7 \mathrm{c}$ & 200000 & $77 \mathrm{~b}$ & $14,3 \mathrm{c}$ & 2507 \\
\hline P 98Y12 RR & 121 & $17,2 \mathrm{~b}$ & 87,3 a & 146667 & $108 \mathrm{~b}$ & $13,7 \mathrm{c}$ & 2293 \\
\hline NA $7200 \mathrm{RR}$ & 118 & $15,2 \mathrm{c}$ & 74,0 a & 236667 & $80 \mathrm{~b}$ & $12,5 \mathrm{c}$ & 2287 \\
\hline NA 5909 RG & 118 & $15,2 \mathrm{c}$ & $61,1 \mathrm{~b}$ & 201667 & $53 \mathrm{~b}$ & $12,1 \mathrm{c}$ & 2187 \\
\hline FPS Iguaçu RR & 118 & $11,9 \mathrm{c}$ & 80,3 a & 133333 & $73 \mathrm{~b}$ & $14,8 \mathrm{c}$ & 2177 \\
\hline $\mathrm{CV}(\%)$ & & 18,68 & 9,59 & & 32,73 & 12,75 & 21,81 \\
\hline
\end{tabular}

Médias seguidas de mesma letra não diferem entre si pelo teste Scott-Knott a 5\% de probabilidade.

Tabela 5. Valores médios da altura de inserção da primeira vagem, altura de plantas, estande final, número de vagens, massa de cem grãos e produtividade de grãos de diferentes cultivares de soja, Araçatuba, SP, safra 2012/13.

\begin{tabular}{|c|c|c|c|c|c|c|}
\hline \multirow[b]{2}{*}{ Cultivar } & \multirow{2}{*}{$\begin{array}{l}\text { Altura de inserção } \\
(\mathrm{cm})\end{array}$} & \multirow[b]{2}{*}{ Altura de plantas $(\mathrm{cm})$} & \multirow{2}{*}{$\begin{array}{l}\text { Estande final } \\
\qquad \mathrm{ha}^{-1}\end{array}$} & \multirow{2}{*}{$\begin{array}{l}\text { Número de vagens } \\
\text { planta }^{-1}\end{array}$} & \multirow{2}{*}{$\begin{array}{l}\text { Massa de } \\
\text { cem grãos }\end{array}$} & \multirow{2}{*}{$\begin{array}{r}\text { (Continua } \\
\text { Produtividade } \\
\left(\mathrm{kg} \mathrm{ha}^{-1}\right)\end{array}$} \\
\hline & & & & & & \\
\hline Anta 82 RR & $16,6 \mathrm{c}$ & $95,8 \mathrm{~b}$ & 422218 & $40,9 \mathrm{~b}$ & $18,5 \mathrm{c}$ & 5293 \\
\hline SYN 1163 RR & $12,0 \mathrm{c}$ & $88,0 \mathrm{~b}$ & 207405 & 88,4 a & $18,4 \mathrm{c}$ & 4978 \\
\hline M-Soy 7908 RR & 22,9 a & $70,1 \mathrm{c}$ & 248146 & 64,3 a & $20,7 \mathrm{c}$ & 4926 \\
\hline
\end{tabular}


Tabela 5. Valores médios da altura de inserção da primeira vagem, altura de plantas, estande final, número de vagens, massa de cem grãos e produtividade de grãos de diferentes cultivares de soja, Araçatuba, SP, safra 2012/13.

(Conclusão)

\begin{tabular}{|c|c|c|c|c|c|c|}
\hline P 97R01 RR & $19,3 \mathrm{~b}$ & 74,8 c & 301849 & 68,2 a & $19,9 \mathrm{c}$ & 4863 \\
\hline INT $6201 \mathrm{RR}$ & $14,7 \mathrm{c}$ & $85,5 \mathrm{~b}$ & 218516 & 67,6 a & $18,9 \mathrm{c}$ & 4841 \\
\hline NS 7490 RR & 23,1 a & 102,9 a & 438885 & $43,4 \mathrm{~b}$ & 17,9 c & 4478 \\
\hline FPS Paranapanema RR & $13,1 \mathrm{c}$ & $65,8 \mathrm{c}$ & 268516 & $48,5 \mathrm{~b}$ & $18,0 \mathrm{c}$ & 3804 \\
\hline INT $6100 \mathrm{RR}$ & $14,8 \mathrm{c}$ & 99,5 a & 361108 & $45,5 \mathrm{~b}$ & $20,3 \mathrm{c}$ & 3785 \\
\hline FPS Iguaçu RR & $16,3 \mathrm{c}$ & $90,8 \mathrm{~b}$ & 420366 & $29,5 \mathrm{~b}$ & $23,6 \mathrm{~b}$ & 3585 \\
\hline $\mathrm{CV}(\%)$ & 11,29 & 8,70 & & 27,03 & 6,39 & 19,06 \\
\hline
\end{tabular}

Médias seguidas de mesma letra não diferem entre si pelo teste Scott-Knott a 5\% de probabilidade.

Tabela 6. Valores médios do florescimento, ciclo, altura de inserção da primeira vagem, altura de plantas, grau de acamamento, estande final, número de vagens e produtividade de grãos de diferentes cultivares de soja, Riolândia, SP, safra 2012/13.

(Continua)

\begin{tabular}{lcccccccc}
\hline Cultivar & $\begin{array}{c}\text { Florescimento } \\
(\text { dias })\end{array}$ & $\begin{array}{c}\text { Ciclo } \\
(\text { dias })\end{array}$ & $\begin{array}{c}\text { Altura de } \\
\text { inserção }(\mathrm{cm})\end{array}$ & $\begin{array}{c}\text { Altura de } \\
\text { plantas }(\mathrm{cm})\end{array}$ & $\begin{array}{c}\text { Acamamento } \\
\text { BRS 232 }\end{array}$ & $\begin{array}{c}\text { Estande } \\
\text { final ha }^{-1}\end{array}$ & $\begin{array}{c}\text { Número de } \\
\text { vagens planta }^{-1}\end{array}$ & $\begin{array}{c}\text { Produtividade } \\
\left(\mathrm{kg} \mathrm{ha}^{-1}\right)\end{array}$ \\
P 98Y11 RR & 48 & 103 & 10,4 & $66,5 \mathrm{~d}$ & $0,0 \mathrm{~d}$ & 418514 & $47 \mathrm{c}$ & 3083 \\
Fundacep 66 RR & 41 & 104 & 12,3 & $104,2 \mathrm{~b}$ & $0,0 \mathrm{~d}$ & 454810 & $34 \mathrm{c}$ & 2814 \\
P 97R01 RR & 45 & 103 & 12,9 & $70,5 \mathrm{~d}$ & $0,0 \mathrm{~d}$ & 311108 & $85 \mathrm{~b}$ & 2778 \\
\hline
\end{tabular}


Tabela 6. Valores médios do florescimento, ciclo, altura de inserção da primeira vagem, altura de plantas, grau de acamamento, estande final, número de vagens e produtividade de grãos de diferentes cultivares de soja, Riolândia, SP, safra 2012/13.

\begin{tabular}{|c|c|c|c|c|c|c|c|c|}
\hline & & & & & & & & (Conclusão) \\
\hline NS 7901 RR & 48 & - & 8,5 & $123,5 \mathrm{a}$ & $3,7 \mathrm{a}$ & 290367 & $96 \mathrm{~b}$ & 2424 \\
\hline NA 5909 RG & 41 & 106 & 12,4 & $87,0 \mathrm{~b}$ & $0,0 \mathrm{~d}$ & 389626 & $63 \mathrm{c}$ & 2385 \\
\hline CD 208 & 42 & 102 & 11,9 & $69,3 \mathrm{~d}$ & $0,0 \mathrm{~d}$ & 348145 & $69 \mathrm{c}$ & 2359 \\
\hline BRS Valiosa RR & 52 & 116 & 16,0 & $92,5 \mathrm{~b}$ & $0,7 \mathrm{c}$ & 264442 & $128 \mathrm{a}$ & 2301 \\
\hline Anta 82 RR & 42 & 105 & 10,7 & $98,5 \mathrm{~b}$ & $0,0 \mathrm{~d}$ & 404440 & $69 \mathrm{c}$ & 2212 \\
\hline BMX Potência RR & 44 & 112 & 10,1 & $93,2 \mathrm{~b}$ & $0,7 \mathrm{c}$ & 285182 & $84 \mathrm{~b}$ & 2210 \\
\hline SYN 9078 RR & 49 & - & 15,7 & 124,5 a & $0,7 \mathrm{c}$ & 268145 & $80 \mathrm{~b}$ & 2167 \\
\hline SYN 1283 RR & 48 & - & 9,1 & $102,8 \mathrm{~b}$ & 4,3 a & 291108 & $61 \mathrm{c}$ & 2163 \\
\hline SYN 1281 RR & 47 & 111 & 15,2 & 130,5 a & $3,7 \mathrm{a}$ & 292590 & $75 \mathrm{c}$ & 2142 \\
\hline INT 6201 RR & 51 & 103 & 11,3 & $90,2 \mathrm{~b}$ & $0,0 \mathrm{~d}$ & 263701 & $71 \mathrm{c}$ & 1964 \\
\hline BRS 284 & 40 & 103 & 14,7 & $96,7 \mathrm{~b}$ & $1,0 \mathrm{c}$ & 379996 & $60 \mathrm{c}$ & 1932 \\
\hline M-Soy 7908 RR & 51 & 110 & 15,5 & $83,6 \mathrm{c}$ & $1,3 \mathrm{c}$ & 282219 & $85 \mathrm{~b}$ & 1844 \\
\hline NS 7255 RR & 50 & 105 & 17,5 & $94,3 \mathrm{~b}$ & $2,3 \mathrm{~b}$ & 290367 & $53 \mathrm{c}$ & 1810 \\
\hline INT 6100 RR & 42 & 107 & 14,4 & $98,9 \mathrm{~b}$ & $0,0 \mathrm{~d}$ & 417774 & $53 \mathrm{c}$ & 1736 \\
\hline FPS Paranapanema RR & 40 & 102 & 10,6 & $60,3 \mathrm{~d}$ & $0,0 \mathrm{~d}$ & 339256 & $64 \mathrm{c}$ & 1643 \\
\hline CV (\%) & & & 30,90 & 8,48 & 74,86 & & 24,73 & 19,23 \\
\hline
\end{tabular}

Médias seguidas de mesma letra não diferem entre si pelo teste Scott-Knott a 5\% de probabilidade. 
Tabela 7. Valores médios do florescimento, ciclo, altura de inserção da primeira vagem, altura de plantas, estande final, número de vagens e produtividade de grãos de diferentes cultivares de soja, Selvíria, MS, safra 2012/13.

(Continua)

\begin{tabular}{|c|c|c|c|c|c|c|c|}
\hline Cultivar & $\begin{array}{c}\text { Florescimento } \\
\text { (dias) }\end{array}$ & $\begin{array}{l}\text { Ciclo } \\
\text { (dias) }\end{array}$ & $\begin{array}{c}\text { Altura de inserção } \\
(\mathrm{cm})\end{array}$ & $\begin{array}{c}\text { Altura de plantas } \\
\text { (cm) }\end{array}$ & $\begin{array}{l}\text { Estande } \\
\text { final ha }{ }^{-1}\end{array}$ & $\begin{array}{c}\text { Número de vagens } \\
\text { planta }^{-1}\end{array}$ & $\begin{array}{c}\text { Produtividade } \\
\left(\mathrm{kg} \mathrm{ha}^{-1}\right)\end{array}$ \\
\hline$\overline{\text { BRS Valiosa RR }}$ & 47 & 128 & $15,0 \mathrm{a}$ & $70,0 \mathrm{~d}$ & 248146 & $110 \mathrm{~b}$ & 4008 \\
\hline M-Soy 7908 RR & 45 & 128 & 16,9 a & $64,8 \mathrm{~d}$ & 276540 & $86 \mathrm{c}$ & 3495 \\
\hline BRS 284 & 30 & 110 & 16,6 a & $88,7 \mathrm{c}$ & 239504 & $66 \mathrm{~d}$ & 3340 \\
\hline P 97R01 RR & 36 & 99 & $15,0 \mathrm{a}$ & $58,7 \mathrm{e}$ & 170369 & $67 \mathrm{~d}$ & 3307 \\
\hline SYN $1281 \mathrm{RR}$ & 38 & 110 & 16,3 a & $97,4 \mathrm{~b}$ & 382712 & $48 \mathrm{~d}$ & 3302 \\
\hline P 98Y11 RR & 41 & 128 & $9,7 \mathrm{~b}$ & $47,1 \mathrm{e}$ & 155554 & $121 \mathrm{~b}$ & 3290 \\
\hline SYN 9078 RR & 41 & 128 & $17,7 \mathrm{a}$ & $100,4 \mathrm{~b}$ & 159258 & $90 \mathrm{c}$ & 3249 \\
\hline NA 7200 RR & 32 & 110 & 15,9 a & $85,3 \mathrm{c}$ & 335799 & $54 \mathrm{~d}$ & 3122 \\
\hline INT $6201 \mathrm{RR}$ & 32 & 110 & $12,5 \mathrm{~b}$ & $82,5 \mathrm{c}$ & 160492 & $79 \mathrm{~d}$ & 3115 \\
\hline FPS Paranapanema RR & 30 & 99 & $8,0 \mathrm{~b}$ & $53,2 \mathrm{e}$ & 185183 & $53 \mathrm{~d}$ & 3110 \\
\hline BRS 232 & 35 & 110 & $11,2 \mathrm{~b}$ & $50,6 \mathrm{e}$ & 212344 & $47 \mathrm{~d}$ & 3108 \\
\hline NS 7901 RR & 38 & 128 & $16,5 \mathrm{a}$ & 109,3 a & 118517 & $169 \mathrm{a}$ & 3026 \\
\hline INT 6100 RR & 33 & 110 & $11,4 \mathrm{~b}$ & $68,5 \mathrm{~d}$ & 266664 & $63 \mathrm{~d}$ & 2952 \\
\hline
\end{tabular}


Tabela 7. Valores médios do florescimento, ciclo, altura de inserção da primeira vagem, altura de plantas, estande final, número de vagens e produtividade de grãos de diferentes cultivares de soja, Selvíria, MS, safra 2012/13.

(Conclusão)

\begin{tabular}{|c|c|c|c|c|c|c|c|}
\hline P 98Y12 RR & 36 & 128 & $16,7 \mathrm{a}$ & $70,4 \mathrm{~d}$ & 199998 & $39 \mathrm{~d}$ & 2945 \\
\hline CD 208 & 32 & 110 & $7,6 \mathrm{~b}$ & $51,5 \mathrm{e}$ & 276540 & $41 \mathrm{~d}$ & 2925 \\
\hline NS 7490 RR & 38 & 110 & $17,7 \mathrm{a}$ & $87,6 \mathrm{c}$ & 219751 & $51 \mathrm{~d}$ & 2818 \\
\hline NA 5909 RG & 32 & 99 & 14,9 a & $74,9 \mathrm{c}$ & 311108 & $49 \mathrm{~d}$ & 2622 \\
\hline SYN 1283 RR & 43 & 128 & $11,4 \mathrm{~b}$ & $75,8 \mathrm{c}$ & 111110 & $92 \mathrm{c}$ & 2299 \\
\hline $\mathrm{CV}(\%)$ & & & 15,70 & 8,12 & & 22,59 & 19,12 \\
\hline
\end{tabular}

Médias seguidas de mesma letra não diferem entre si pelo teste Scott-Knott a 5\% de probabilidade.

Tabela 8. Valores médios do florescimento, ciclo, altura de inserção da primeira vagem, altura de plantas, estande final, número de vagens, massa de cem grãos e produtividade de grãos de diferentes cultivares de soja, Votuporanga, SP, safra 2012/13.

\begin{tabular}{|c|c|c|c|c|c|c|c|c|}
\hline Cultivar & $\begin{array}{c}\text { Florescimento } \\
\text { (dias) }\end{array}$ & $\begin{array}{l}\text { Ciclo } \\
\text { (dias) }\end{array}$ & $\begin{array}{c}\text { Altura de } \\
\text { inserção }(\mathrm{cm})\end{array}$ & $\begin{array}{c}\text { Altura de } \\
\text { plantas }(\mathrm{cm})\end{array}$ & $\begin{array}{c}\text { Estande final } \\
\mathrm{ha}^{-1}\end{array}$ & $\begin{array}{c}\text { Número de } \\
\text { vagens planta }^{-1}\end{array}$ & $\begin{array}{l}\text { Massa de } \\
\text { cem grãos }\end{array}$ & $\begin{array}{l}\text { Produtividade } \\
\qquad\left(\mathrm{kg} \mathrm{ha}^{-1}\right)\end{array}$ \\
\hline NS 7901 RR & 44 & 118 & 18,0 a & 86,5 a & 216000 & 83 & $15,2 \mathrm{~d}$ & 4143 \\
\hline NS 7255 RR & 40 & 118 & $12,8 \mathrm{~b}$ & $73,6 \mathrm{~b}$ & 252000 & 34 & $13,8 \mathrm{e}$ & 3591 \\
\hline P 98Y12 RR & 45 & 118 & $18,2 \mathrm{a}$ & $74,6 \mathrm{~b}$ & 232000 & 44 & $16,5 \mathrm{c}$ & 3554 \\
\hline
\end{tabular}


Tabela 8. Valores médios do florescimento, ciclo, altura de inserção da primeira vagem, altura de plantas, estande final, número de vagens, massa de cem grãos e produtividade de grãos de diferentes cultivares de soja, Votuporanga, SP, safra 2012/13.

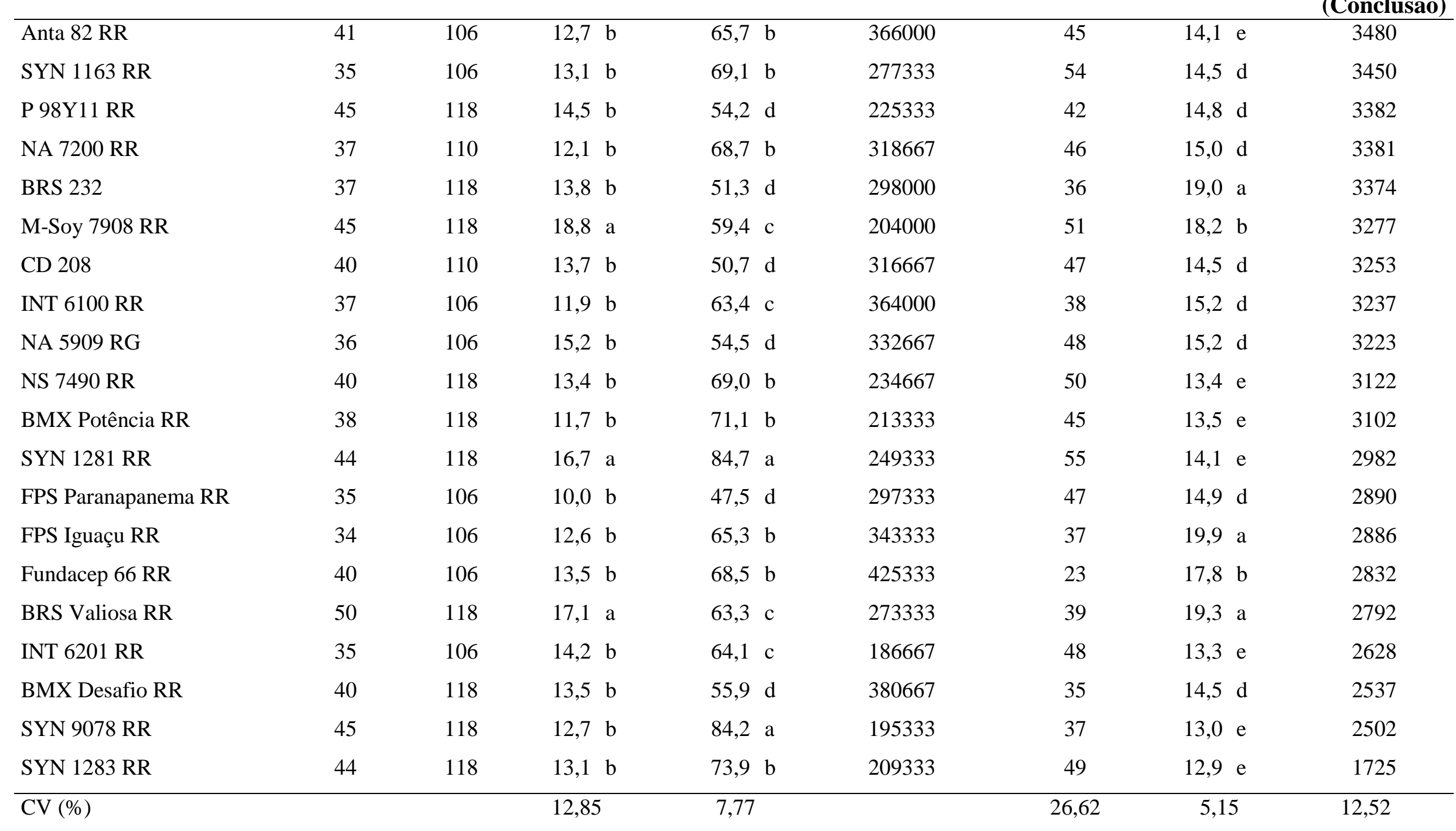

Médias seguidas de mesma letra não diferem entre si pelo teste Scott-Knott a 5\% de probabilidade. 
Tabela 9. Análise conjunta dos valores médios da altura de inserção da primeira vagem, altura de plantas, estande final, número de vagens e produtividade de grãos de diferentes cultivares de soja, safra 2011/12.

\begin{tabular}{|c|c|c|c|c|c|c|c|}
\hline Cultivar & $\begin{array}{c}\text { Altura de } \\
\text { inserção }(\mathrm{cm})\end{array}$ & $\begin{array}{l}\text { Altura de plantas } \\
\qquad(\mathrm{cm})\end{array}$ & Acamamento & $\begin{array}{l}\text { Estande } \\
\text { final ha }{ }^{-1}\end{array}$ & $\begin{array}{l}\text { Número de vagens } \\
\text { planta }^{-1}\end{array}$ & $\begin{array}{l}\text { Massa de cem } \\
\text { grãos }\end{array}$ & $\begin{array}{l}\text { Produtividade } \\
\qquad\left(\mathrm{kg} \mathrm{ha}^{-1}\right)\end{array}$ \\
\hline$\overline{C D} 208$ & 12,2 & $61,9 \mathrm{c}$ & 0,3 & 265158 & $54 \mathrm{a}$ & $14,0 \mathrm{~b}$ & 3943 \\
\hline SYN 2395 RR & 12,8 & $66,4 \mathrm{c}$ & 0,3 & 385105 & $42 \mathrm{~b}$ & 15,6 a & 3503 \\
\hline NS 7100 RR & 14,7 & $76,7 \mathrm{~b}$ & 0,3 & 336219 & $42 \mathrm{~b}$ & 15,6 a & 3488 \\
\hline NA 5909 RG & 14,2 & $59,5 \mathrm{c}$ & 0,3 & 323371 & $34 \mathrm{~b}$ & 15,6 a & 3258 \\
\hline RA 518 RR & 12,2 & $55,0 \mathrm{c}$ & 0,3 & 274722 & $34 \mathrm{~b}$ & 16,4 a & 3177 \\
\hline M-Soy 7908 RR & 18,4 & $67,6 \mathrm{c}$ & 0,3 & 235183 & $46 \mathrm{~b}$ & 16,6 a & 3148 \\
\hline SYN 1080 RR & 15,2 & $65,9 \mathrm{c}$ & 0,7 & 312573 & $32 \mathrm{~b}$ & $14,5 \mathrm{~b}$ & 2903 \\
\hline
\end{tabular}

Médias seguidas de mesma letra não diferem entre si pelo teste Scott-Knott a 5\% de probabilidade.

Tabela 10. Análise conjunta dos valores médios da altura de inserção da primeira vagem, altura de plantas, estande final, número de vagens e produtividade de grãos de diferentes cultivares de soja, safra 2012/13.

(Continua)

\begin{tabular}{|c|c|c|c|c|c|}
\hline Cultivar & Altura de inserção $(\mathrm{cm})$ & Altura de plantas $(\mathrm{cm})$ & Estande final ha $^{-1}$ & Número de vagens planta $^{-1}$ & Produtividade $\left(\mathrm{kg} \mathrm{ha}^{-1}\right)$ \\
\hline NS 7901 RR & $15 \mathrm{~b}$ & $103 \mathrm{a}$ & 196221 & $126 \mathrm{a}$ & 3172 \\
\hline BRS Valiosa RR & $17 \mathrm{a}$ & $76 \mathrm{~b}$ & 227730 & $111 \mathrm{a}$ & 3128 \\
\hline BRS 232 & $11 \mathrm{c}$ & $55 \mathrm{~d}$ & 269576 & $53 \mathrm{c}$ & 3100 \\
\hline P 98Y11 RR & $12 \mathrm{c}$ & $62 \mathrm{~d}$ & 197443 & $84 \mathrm{~b}$ & 3024 \\
\hline
\end{tabular}


Tabela 10. Análise conjunta dos valores médios da altura de inserção da primeira vagem, altura de plantas, estande final, número de vagens e produtividade de grãos de diferentes cultivares de soja, safra 2012/13.

(Conclusão)

\begin{tabular}{|c|c|c|c|c|c|}
\hline P 97R01 RR & $15 \mathrm{~b}$ & $62 \mathrm{~d}$ & 217703 & $69 \mathrm{~b}$ & 3020 \\
\hline Anta 82 RR & $13 \mathrm{c}$ & $80 \mathrm{~b}$ & 305526 & $55 \mathrm{c}$ & 2986 \\
\hline M-Soy 7908 RR & $18 \mathrm{a}$ & $71 \mathrm{c}$ & 221107 & $76 \mathrm{~b}$ & 2977 \\
\hline BRS 284 & $14 \mathrm{~b}$ & $81 \mathrm{~b}$ & 265708 & $71 \mathrm{~b}$ & 2961 \\
\hline SYN 1163 RR & $13 \mathrm{c}$ & $86 \mathrm{~b}$ & 235551 & $77 \mathrm{~b}$ & 2951 \\
\hline CD 208 & $12 \mathrm{c}$ & $60 \mathrm{~d}$ & 277005 & $63 \mathrm{c}$ & 2901 \\
\hline Fundacep 66 RR & $14 \mathrm{~b}$ & $84 \mathrm{~b}$ & 337998 & $37 \mathrm{c}$ & 2867 \\
\hline SYN 1281 RR & $19 \mathrm{a}$ & 99 a & 336575 & $54 \mathrm{c}$ & 2836 \\
\hline NS 7255 RR & $15 \mathrm{~b}$ & $81 \mathrm{~b}$ & 235560 & $52 \mathrm{c}$ & 2822 \\
\hline P $98 Y 12$ RR & $16 \mathrm{~b}$ & $82 \mathrm{~b}$ & 219665 & $72 \mathrm{~b}$ & 2800 \\
\hline BMX Potência RR & $11 \mathrm{c}$ & $83 \mathrm{~b}$ & 202807 & $79 \mathrm{~b}$ & 2774 \\
\hline NA 7200 RR & $14 \mathrm{c}$ & $82 \mathrm{~b}$ & 307967 & $65 \mathrm{~b}$ & 2720 \\
\hline NS 7490 RR & $14 \mathrm{~b}$ & $85 \mathrm{~b}$ & 230178 & $65 \mathrm{~b}$ & 2693 \\
\hline INT $6100 \mathrm{RR}$ & $12 \mathrm{c}$ & $76 \mathrm{~b}$ & 288359 & $61 \mathrm{c}$ & 2651 \\
\hline NA 5909 RR & $14 \mathrm{~b}$ & $69 \mathrm{c}$ & 308767 & $53 \mathrm{c}$ & 2604 \\
\hline BMX Desafio RR & $13 \mathrm{c}$ & $77 \mathrm{~b}$ & 337109 & $58 \mathrm{c}$ & 2566 \\
\hline INT $6201 \mathrm{RR}$ & $12 \mathrm{c}$ & $78 \mathrm{~b}$ & 176465 & $99 \mathrm{a}$ & 2538 \\
\hline FPS Paranapanema RR & $10 \mathrm{c}$ & $54 \mathrm{~d}$ & 255443 & $60 \mathrm{c}$ & 2537 \\
\hline FPS Iguaçu RR & $13 \mathrm{c}$ & $80 \mathrm{~b}$ & 261943 & $49 \mathrm{c}$ & 2326 \\
\hline NS 7901 RR & $15 \mathrm{~b}$ & $103 \mathrm{a}$ & 196221 & $126 \mathrm{a}$ & 3172 \\
\hline BRS Valiosa RR & $17 \mathrm{a}$ & $76 \mathrm{~b}$ & 227730 & $111 \mathrm{a}$ & 3128 \\
\hline$\overline{\mathrm{CV}}(\%)$ & 23,97 & 17,83 & & 44,91 & 23,18 \\
\hline
\end{tabular}

Médias seguidas de mesma letra não diferem entre si pelo teste Scott-Knott a 5\% de probabilidade. 


\section{AGRADECIMENTOS}

Agradecemos a Syngenta, Dupont Pioneer e Nidera Sementes pelo apoio ao projeto que originou este trabalho, na safra 2012/13.

Agradecemos também a todos os funcionários e estagiários dos Polos Regionais de Desenvolvimento Tecnológico dos Agronegócios do Noroeste Paulista e Extremo Oeste - APTA e do EDR e DSMM/NPS de Araçatuba - CATI, pelo apoio na condução dos ensaios.

\section{CONCLUSÕES}

É necessária a continuação dos estudos para melhor recomendação das cultivares mais adaptadas a Região Noroeste Paulista.

\section{REFERÊNCIAS}

ARAUJO JUNIOR, O.; ANDRADE, E. A.; HAYASHI, F. K.; LAZARINI, E.; FERREIRA, M.B.; SOUZA, L. G. M. Avaliação de cultivares de soja em duas densidades no município de Selvíria - MS Safra 2010/11. CONGRESSO BRASILEIRO DE SOJA, 6., 2012, Cuiabá. Anais... Londrina: Embrapa Soja, 2012.

BERTAGNOLLI, P. F.et al.. Rendimento de grãos de cultivares de soja tolerantes a glifosato, Rede Soja Sul de Pesquisa, safra 2010/11 - Macrorregião Sojícola 1. CONGRESSO BRASILEIRO DE SOJA, 6., 2012, Cuiabá. Anais... Londrina: Embrapa Soja, 2012.

BIGHI, W.et al. O. Comportamento de genótipos de soja (Glycine max (L.) Merrill) cultivados em Jaboticabal/SP e Ipameri/GO, ano agrícola 2002/2003. Ceres, Viçosa, v. 53, n. 310, p. 682-687, 2006.

COMPANHIA NACIONAL DE ABASTECIMENTO - CONAB. Séries históricas relativas às safras 1976/77 a 2012/2013 de área plantada, produtividade e produção. Brasília, DF, 2013. (Série histórica). Disponível em:

<http://www.conab.gov.br/conteudos.php?a=1252\&t=\&Pagina_objcmsconteudos=3\#A_objcmsconteudos >. Acesso em: 20 maio 2013.

CRUZ, T. V.et al. Componentes de produção de soja em diferentes épocas de semeadura, no Oeste da Bahia. Bioscience Journal, Uberlândia, v. 26, n. 5, p. 709-716, 2010.

EMBRAPA. Tecnologias de produção de soja - região central do Brasil 2012 e 2013. Londrina: Embrapa Soja, 2011. p. 95-116. (Sistemas de Produção / Embrapa Soja, n. 15)

FUNDAÇÃO MT. Boletim de Pesquisa de Soja. Rondonópolis: Fundação de Apoio a Pesquisa Agropecuária de Mato Grosso - Fundação MT, 2007. P. 63-128. (Boletim de Pesquisa de Soja, n.11)

HAYASHI, F.K.et al. Comportamento de variedades de soja em Selvíria - MS - Safra 2010/11. CONGRESSO BRASILEIRO DE SOJA, 6., 2012, Cuiabá. Anais... Londrina: Embrapa Soja, 2012.

HERNANDEZ, F. B. T., LEMOS FILHO, M. A. F.; BUZETTI, S. Software HIDRISA e o balanço hídrico de Ilha Solteira. Ilha Solteira: UNESP/FEIS, 1995. 45p. (Área de Hidráulica e Irrigação. Série Irrigação, 1)

KOMORI, E.et al. Influencia da época de semeadura e população de plantas sobre as características agronômicas da cultura da soja. Bioscience Journal, Uberlândia, v. 20, n. 3, p. 13-p14, 2004. 
MAUAD, M.et al. Influência da densidade de semeadura sobre características agronômicas na cultura da soja. Agrarian, Dourados, v. 3, n. 9, p. 175-181, 2010.

PEIXOTO, C. P.et al.. Épocas de semeadura e densidades de plantas de soja: I. Componentes da produção e rendimento de grãos. Scientia Agrícola, Piracicaba, v. 57, n. 1, p. 89-96, 2000.

PEREIRA, A. O mundo dependerá mais da soja do Cone Sul na safra 2012/13. In: Agrianual (2013) Anuário da agricultura brasileira. São Paulo: AGRA FNP Pesquisas Ltda., 2012, p. 417-422.

PELUZIO, J. M.et al.. Desempenho de cultivares de soja na Região Centro-Sul do Estado do Tocantins SAFRA 2007/08. Bioscience Journal, Uberlândia, v. 26, n. 5, p. 675-682, 2010.

SOARES, I. O. et al.. Seleção de cultivares de soja em cultivo de verão para o sul de Minas Gerais. CONGRESSO BRASILEIRO DE SOJA, 6., 2012, Cuiabá. Anais... Londrina: Embrapa Soja, 2012.

YUYAMA, K. Avaliação de algumas características agronômicas e morfofisiológicas de cinco cultivares de soja (Glycine max (L.) Merril), cultivados em solo de várzea e de terra firme da Amazônia Central. 1991. 123 f. Tese (Doutorado em Produção Vegetal) - Faculdade de Ciências Agrárias e Veterinárias, Universidade Estadual Paulista, Jaboticabal, 1991. 
